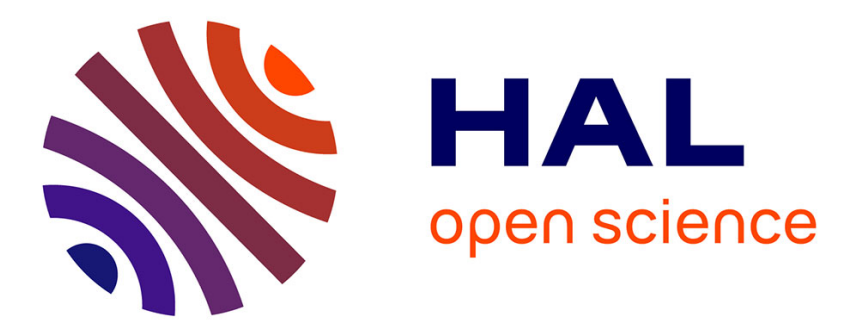

\title{
Differential reactions of virtual actors and observers to the triggering and interruption of psychological momentum
}

Walid Briki, Julie Doron, Keith D. Markman, Ruud J. R den Hartigh, Christophe Gernigon

\section{To cite this version:}

Walid Briki, Julie Doron, Keith D. Markman, Ruud J. R den Hartigh, Christophe Gernigon. Differential reactions of virtual actors and observers to the triggering and interruption of psychological momentum. Motivation and Emotion, 2013, 10.1007/s11031-013-9372-3 . hal-01579148

\section{HAL Id: hal-01579148 \\ https://hal-insep.archives-ouvertes.fr/hal-01579148}

Submitted on 30 Aug 2017

HAL is a multi-disciplinary open access archive for the deposit and dissemination of scientific research documents, whether they are published or not. The documents may come from teaching and research institutions in France or abroad, or from public or private research centers.
L'archive ouverte pluridisciplinaire HAL, est destinée au dépôt et à la diffusion de documents scientifiques de niveau recherche, publiés ou non, émanant des établissements d'enseignement et de recherche français ou étrangers, des laboratoires publics ou privés. 


\title{
Differential reactions of virtual actors and observers to the triggering and interruption of psychological momentum
}

\author{
Walid Briki · Julie Doron · Keith D. Markman · \\ Ruud J. R. Den Hartigh · Christophe Gernigon
}

Abstract The present study compared virtual actors' and observers' perceptions of positive and negative psychological momentum (PM) and their responses to sudden interruptions of momentum. Participants with experience playing competitive table tennis imagined that they were playing a table tennis game (virtual actors), whereas participants who lacked experience playing competitive table tennis imagined that they were observing a table tennis game (observers). While viewing an audiovisual simulation of a table tennis match, participants were exposed to either an ascending (i.e., positive momentum) or descending (i.e., negative momentum) scoring sequence that was either

The terms momentum and psychological momentum $(P M)$ are often used interchangeably in the psychological literature, which may elicit some confusion. To be precise, the term "PM" is intended to describe the emotional experience evoked by the sense that "everything is going right" (positive PM) or that "everything is going wrong", (negative PM), whereas "momentum" is intended to describe the contextual factors that evoke "PM." Consequently, the present article uses the term "PM" to specify the emotional experiences induced by momentum sequences.

W. Briki (\&) • R. J. R. Den Hartigh • C. Gernigon Department of Sport and Physical Education Sciences, Southern France Montpellier University, 700 Avenue du Pic St Loup, 34090 Montpellier, France

e-mail: briki.w@gmail.com

J. Doron

Department of Sport and Physical Education Sciences, ParisEast Créteil University, Paris, France

K. D. Markman

Department of Psychology, Ohio University, Athens, OH, USA

R. J. R. Den Hartigh

Department of Psychology, University of Groningen, Groningen, The Netherlands suddenly interrupted or not interrupted at all. Participants' PM perceptions were measured at the conclusion of the simulation. Results indicated that observers' PM perceptions were lower than were virtual actors' following the negative momentum sequence. More generally, interrupting positive momentum lowered PM perceptions, whereas interrupting negative momentum increased PM perceptions. Implications for the study of PM in sport are discussed.

Keywords Actors $\cdot$ Inertia $\cdot$ Observers $\cdot$

Pathetic fallacy $\cdot$ Performance

\section{Introduction}

Psychological Momentum (PM) is commonly regarded as an acquired psychological force that runs through many life domains (Adler 1981). PM is a bidirectional phenomenon: While experiencing positive PM, everything seems to be going smoothly, while when experiencing negative PM, everything seems to be going wrong. Every conceptualization of PM in the literature stresses that this phenomenon includes a number of psychological features-related to perceptual, cognitive, affective, motivational and behavioral spheres - that shift according to perceptions of progress in relation to a goal to be attained (Adler 1981; Gernigon et al. 2010; Markman and Guenther 2007; Taylor and Demick 1994; Vallerand et al. 1988). The perception of movement toward or away from a goal is thought to arise from a precipitating event or series of events that occurs within a context of importance and that provides an initial impetus (Taylor and Demick 1994; Markman and Guenther 2007). Adler (1981) considered that such an impetus may develop either suddenly (explosive $P M$ ) or 
gradually (placid PM), and that explosive PM would be experienced more intensely but also be more susceptible to interruption than would placid PM. An impetus (as well as an interruption) can be felt by actors who are directly involved in an event (e.g., Briki et al. 2012a, b; Briki et al. 2013; Perreault et al. 1998; Stanimirovic and Hanrahan, 2004) as well as observers of the very same event (e.g., Markman and Guenther 2007; Vallerand et al. 1988). The present research examines how actors and observers perceive PM and how a sudden interruption of a momentum sequence may impact their subjective experience of PM.

\section{How actors and observers perceive PM}

Multiple studies have demonstrated the effects of repeated successes or failures on PM perceptions (e.g., Eisler and Spink 1998; Kerick et al. 2000; Markman and Guenther 2007; Miller and Weinberg 1991; Perreault et al. 1998; Shaw et al. 1992; Silva et al. 1992; Stanimirovic and Hanrahan 2004; Vallerand et al. 1988). While some studies have employed actors responding during an actual sports competition (e.g., Kerick et al. 2000; Perreault et al. 1998; Shaw et al. 1992; Stanimirovic and Hanrahan 2004), others have employed observers of sports competitions, or, study participants responding to hypothetical scenarios (e.g., Markman and Guenther 2007; Vallerand et al. 1988). According to Vallerand et al. (1988), the more an individual is involved in the action, the more he or she will experience cognitions, affects, and motivations related to PM. In other words, Vallerand et al. (1988) hypothesized that task involvement and PM perception intensity should be positively related. To date, however, this assumption remains untested.

Outside the domain of PM, Deutsch (1960) explored differences between the emotional experiences of actors' and observers' estimations of actors' emotional experiences. In two experiments, Deutsch (1960) asked telephone operators to individually perform a team-relevant task, after which they received either a favorable or unfavorable evaluation of their performance that was supposedly provided by a team member. Other participants who were just observing the completion of the task were asked to assess how the actors perceived the evaluation of their performance. Results indicated that the observers perceived the favorable evaluation more positively than did the actors themselves, and that they also viewed the unfavorable evaluation more negatively than did the actors. Deutsch (1960) explained this polarization of observers' ratings by employing the concept of the pathetic fallacy, which refers to a general tendency to evaluate others in terms of successes and failures (accompanied by the social injunction to appear successful). The concept of the pathetic fallacy is based on the assumption that in social interactions, individuals develop expectations-rooted in dominant social values and their personal experiences in societyabout what others are like and what their behaviors suggest (see also Goffman 1955). Thus, in a culture that emphasizes achievement, such as in Western culture, any success or failure is expected to impact the actor's experience strongly (Deutsch 1960).

Notably, whereas Vallerand et al. (1988) assumed that observers should perceive PM less intensely than should actors themselves, Deutsch's (1960) findings suggest that observers should actually perceive PM more intensely than actors. Hence, a major purpose of the present study was to explore how perceptions of PM differ among actors and observers.

\section{The effects of interruption on PM}

Recently, Markman and Guenther (2007) examined the construct of inertia- a dynamical feature of PM perception. The authors hypothesized that PM is perceived as an enduring impetus that is transferable to subsequent tasks. Naïve theories about this inertial property were examined in an experiment (Experiment 3) where participants either read a scenario in which a woman began cleaning her apartment at a moderate pace before "getting on a cleaning roll" (i.e., momentum condition), or a scenario in which a woman cleaned her apartment at a steady pace (i.e., no momentum condition). Relative to participants in the no momentum condition, participants in the momentum condition perceived that after cleaning her apartment the woman would be better able to make further progress on a paper that she needed to write for a class assignment. Additional evidence for the property of inertia was found by Markman and Guenther (2007) in a subsequent experiment (Experiment 4) in which participants read a scenario about a female student who was either making steady progress (slowly progressing toward her goal) or momentous progress (quickly progressing toward her goal after not having made any progress) on a class writing assignment (with objective progress held constant across both scenarios). Following a sudden and unexpected interruption (a phone call from her mother), participants believed that it would be more difficult for the student to complete her paper by a deadline when she was interrupted while making momentous progress than when she was interrupted while making steady progress. In other words, participants assumed that PM would be difficult to regain after it was interrupted.

Markman and Guenther's (2007) findings suggest an analogy between PM and Newtonian physics principles because PM can be perceived as both an impetus that carries over to subsequent situations as well as an impetus that is difficult to regain once interrupted. However, their 
experimental paradigm was limited in two important ways. First, PM was manipulated by qualitatively described scenarios and thus not in a directly quantitative way that delineated a clear and discrete progression toward a desired goal. Second, these authors focused only on positive PM. Addressing these limitations, the present study focused on the effects of a sudden interruption of both positive and negative momentum sequences on PM perceptions by employing a more engaging audiovisual simulation of a sports competition.

\section{Goals of the present research}

Using an audiovisual simulation of a table tennis match, the present research tested whether virtual actors and observers react differently to the triggering and the interruption of PM. The first purpose was to test Vallerand et al. (1988) assumption that actors and observers view PM differently. Whereas Vallerand et al. (1988) suggested (but presented no empirical data indicating) that observers should react less strongly to momentum-evoking situations than should actors, Deutsch (1960) found that observers perceive actors' feelings more strongly than do the actors themselves. In line with Deutsch's finding, it was predicted in the present study that participants viewing an audiovisual simulation from the point of view of an observer would perceive PM more intensely than would participants viewing the simulation from the perspective of a virtual actor. The second purpose of this research was to test Markman and Guenther's (2007) view that PM is an impetus that is experienced until some event causes one to lose it. To this end, the impact of a sudden interruption of both positive and negative momentum sequences on PM perceptions was examined. Consistent with Markman and Guenther (2007), it was predicted that an interruption of positive momentum would diminish PM perceptions and, extending Markman and Guenther (2007), it was also predicted that an interruption of negative momentum would increase PM perceptions. Moreover, we explored whether the effects of momentum interruption on PM perceptions are moderated by involvement type-virtual actors versus observers.

\section{Method}

\section{Participants}

The study employed a sample of table tennis players with prior competitive experience (virtual actors) and a separate sample of individuals who had no prior competitive experience playing table tennis (observers). The experienced sample included 110 table tennis players who were recruited from several clubs in the south and west of France with the consent of their presidents and coaches, and who were presently competing at a departmental (13 males, 0 female; $\left.M_{\text {age }}=34.3, S D_{\text {age }}=14.8\right)$, regional (53 males, 16 females; $\left.M_{\text {age }}=27.2, S D_{\text {age }}=10.8\right)$ or national level $(25$ males, 3 females; $\left.M_{\text {age }}=26.1, S D_{\text {age }}=11\right)$. On average they had been participating for 7.1 years $(S D=5.5)$ in their competition level. The non-experienced sample included 117 students in the sports sciences who had prior experience participating in sports competitions but had never participated in a table tennis competition.

Only participants who reported in the manipulation check (see the Measures section below) that they were able to project themselves into the audiovisual simulation were included in the final virtual actor and observer samples. The two final samples comprised 84 virtual actors (71 males, 13 females; $11.9 \%$ departmental level players, $67.6 \%$ regional level players, and $20.2 \%$ national level players; $M_{\text {age }}=28.6$, $\left.S D_{\text {age }}=12.0\right)$ and 83 observers (55 males, 28 females; $\left.M_{\text {age }}=22.5, S D_{\text {age }}=3.7\right)$. Participants $(N=167)$ were assigned to groups corresponding to the eight experimental conditions of a 29292 between-subjects design (Involvement Type: virtual actors vs. observers 9 Momentum Sequence: positive vs. negative 9 Interruption: interruption vs. no interruption). Within each level of Involvement Type, the assignment of participants to the different conditions of Momentum Sequence and Interruption was carried out randomly.

\section{Materials and design}

Table tennis was chosen as a study context because in this sport performance yields numerically represented points that unfold through discrete exchanges, lending itself well for a momentum manipulation. Virtual simulations were used to standardize the experimental conditions. The experimental setup included a computer installed in a room at the university. On the computer screen participants viewed an audiovisual sequence created in Microsoft PowerPoint (Office 2007). A grey slide continuously displayed the scores of the two players. The score on the left side of the screen was colored blue and corresponded to the score of the target player, whereas the score on the right side of the screen was colored green and corresponded to the score of the target player's opponent. The scores changed each time the participant heard an audio clip of a table tennis rally. The clip started with a silent period preceding the service, followed by the sounds of the service and several exchanges between the players. The end of the clip consisted of the clapping sounds of spectators. 


\section{Momentum conditions}

Because PM develops more intensely when the situational goal is of higher relevance to the individual (Markman and Guenther 2007), the manipulated momentum sequences focused upon the decisive game of the match (the fifth game). The positive and negative momentum sequences began at scores of $0-7$ and 7-0, respectively, and both ended at a score of 7-7. Thus, in the positive momentum sequence, the target player gradually came from behind by winning seven points in a row, whereas in the negative momentum sequence the target player began with a lead but then lost seven points in row.

\section{Interruption conditions}

In the interruption condition, the interruption was conveyed by another audio clip that was played when the score was 7-7. This clip included two phases: A silent pause, followed by the sounds of a power cut and the "aahs!" of disappointed spectators. The choice to simulate a loss of power as a momentum interruption was based on a national level table tennis coach's suggestion: A power cut, lying outside the control of the players, is an event that might plausibly occur during a table tennis match. Directly after the power cut, a black slide appeared on which the following message was displayed for $30 \mathrm{~s}$ : "There is a power cut in the gym. Please wait'. Subsequently, a second black slide appeared for $30 \mathrm{~s}$, which read: "Currently, technicians are trying to solve the problem. Please wait'. Finally, a grey slide appeared, displaying the following text: "Things are ok now, the problem is solved. Please wait", after which (30 s later) the final score of 7-7 was again displayed. In total, the interruption lasted $1.5 \mathrm{~min}$. In the no interruption condition, on the other hand, the scenario presentation simply ended with the score tied at 7-7, with no subsequent description of a power loss.

\section{Procedure}

Participants signed an informed consent form upon their arrival in the experimental room. They were assured that their responses would remain confidential. Virtual actors then played the role of table tennis players by imagining that they were actually playing. To this purpose, they received the following instructions:

Now, imagine that you are a table tennis player, and you are competing against another player in the most important competition of your career. You are in the final round, and in this final the match is tied: Both you and your opponent have won two games. Thus, this is the moment when the decisive game will be played, and in this extremely important game, you are 7-0 (or 0-7) ahead (or behind).

Observers, on the other hand, played the role of fans of one of the two table tennis players depicted in the simulation. They therefore received the following instructions:

Now, imagine that you are a table tennis fan and that you are supporting your favorite player who is competing against another player in the most important competition of his career. Hence, this competition is also extremely important to you. In this competition, your player is in the final round, and in this final the match is tied: Both your player and his opponent have won two games. Thus, this is the moment when the decisive game will be played, and in this extremely important game for your player, and hence for you as a fan, your player is 7-0 (0-7) ahead (or behind).

After receiving these instructions, participants imagined the match situation. To help them, they listened to (with headphones) and watched (on the screen where the score evolvement was displayed) the table tennis rallies according to the conditions of positive or negative momentum sequences that were interrupted or not. When the simulation was finished, participants responded to the manipulation check and PM perception items. The experimenter remained in the room during the entire procedure.

Measures

\section{PM perceptions}

Psychological momentum perceptions were assessed with six items that were obtained from Vallerand et al. (1988) "perceptions of PM" questionnaire that underwent a backtranslation procedure (Brislin et al. 1973), and were adapted when needed to fit with typical French expressions reflecting PM. Accordingly, the questions were the following: "Who seems to have the most wind on one's sails?"; "Who seems to be the most confident?"; "Who seems to have the most control over the game?"; "Who seems to be the most motivated?"; "Who seems to be the most discouraged?" (reverse-coded item); and "Who seems to progress the most toward victory?". Virtual actors were asked to answer each question on a 7-point Likerttype scale ranging from 1 ("certainly my opponent") to 7 ("certainly me"), with a neutral midpoint 4 ("neither me nor my opponent"). Observers were asked to make ratings along the same scale type ranging from 1 ("certainly my player's opponent") to 7 ("certainly my player"), with a neutral midpoint 4 ("neither my player nor my player's opponent"). The dependent variable was the state of perceived PM that was accounted for by the average of the 
scores across all six items. The six items were highly internally consistent $(\mathrm{a}=.93)$.

\section{Manipulation check}

At the end of the experiment, participants also answered a question that measured their degree of immersion in the task. Virtual actors responded on a 7-point Likert-type scale ranging from 1 ("not at all") to 7 ("very much so") to the question: "To what extent did you succeed in imagining yourself as being the player you were supposed to be in the decisive game of the final of the most important competition of your career?". Likewise, observers responded on the same scale to the question: "To what extent did you succeed in imagining yourself as encouraging the player for whom you were supposed to be a fan in the decisive game of the final of the most important competition of your player's career?" Data from participants who responded at or below the middle of the scale (i.e., 26 virtual actors and 34 observers) were excluded from the analyses. This selection was made in order to ensure that participants whose data were analyzed all cooperated with the instruction to either imagine that they were a player, or to empathize with a player.

Data analysis

To examine our specific hypotheses regarding the interactions between Momentum Sequence and Involvement Type and between Momentum Sequence and Interruption, a 29292 ANOVA (Involvement Type 9 Momentum Sequence 9 Interruption) was processed on PM perceptions. Follow-up post hoc comparisons (Scheffé tests) were applied in order to compare means among the conditions. Scheffé tests were employed because they are the most statistically conservative (i.e., the least statistically powerful) post hoc comparison procedure, thereby enhancing the reliability and validity of any statistically significant comparisons that might be found. All Levene's tests were non-significant ( $p \backslash .05$ ), thereby supporting the assumption of homogeneous sample variances.

\section{Results}

The means and standard deviations of PM perceptions for each condition are presented in Table 1, and corresponding graphs of the means are displayed in Fig. 1. The 29292 ANOVA (Involvement Type 9 Momentum Sequence 9 Interruption) that was conducted on PM perceptions revealed a significant main effect of Momentum Sequence $\left(F_{(1,159)}=584.58, p \backslash .001\right.$, partial $\left.\mathrm{g}^{2}=.79\right)$, a significant Involvement Type 9 Momentum Sequence interaction effect $\left(F_{(1,159)}=5.39, p \backslash .05\right.$, partial $\left.^{2}=.03\right)$,
Table 1 Descriptive statistics of PM perceptions according to involvement type, momentum sequence, and interruption

\begin{tabular}{llll}
\hline & $\begin{array}{l}\text { Actor } \\
\text { M (SD) }\end{array}$ & $\begin{array}{l}\text { Observer } \\
\text { M (SD) }\end{array}$ & $\begin{array}{l}\text { All Participants } \\
\text { M (SD) }\end{array}$ \\
\hline $\begin{array}{l}\text { Positive momentum } \\
\text { No interruption }\end{array}$ & $6.17(0.70)$ & $5.83(0.76)$ & $6.01(0.74)$ \\
Interruption & $5.29(0.75)$ & $5.72(0.72)$ & $5.51(0.75)$ \\
All & $5.74(0.84)$ & $5.78(0.72)$ & $5.76(0.78)$ \\
Negative momentum & & & \\
No interruption & $2.83(0.93)$ & $2.16(0.56)$ & $2.48(0.83)$ \\
Interruption & $3.21(0.96)$ & $2.82(0.97)$ & $3.02(0.97)$ \\
All & $3.02(0.95)$ & $2.49(0.85)$ & $2.75(0.96)$ \\
\hline
\end{tabular}

and a significant Momentum Sequence 9 Interruption interaction effect $\left(F_{(1,159)}=16.79, p \backslash .001\right.$, partial $g^{2}=$ $.10)$. No significant main effects were found for Involvement Type or for Interruption, and neither the Involvement Type 9 Interruption interaction nor the omnibus Involvement Type 9 Momentum Sequence 9 Interruption interaction were significant ( $p s$ [.05).

The means in Table 1 indicate that PM perceptions were higher following the positive momentum sequence $(M=5.76)$ than following the negative momentum sequence $(M=2.75)$. Moreover, Scheffé tests revealed that observers following the negative momentum sequence had lower PM perceptions than did: (a) observers following the positive momentum sequence $(p \backslash .001)$ and (b) virtual actors following the negative momentum sequence $(p \backslash .05)$. Virtual actors' perceptions of PM were lower following the negative momentum sequence than following the positive momentum sequence ( $p \backslash .001)$. However, no significant difference was found between virtual actors and observers following the positive momentum sequence $(p$ 【.05). For both the interruption and the no interruption conditions, PM perceptions were significantly higher following the positive momentum sequence than following the negative momentum sequence $(p \backslash .001)$. Finally, and consistent with predictions, following the negative momentum sequence PM perceptions were significantly higher in the interruption condition than in the no interruption condition ( $p \backslash .05)$, whereas the contrary was observed following the positive momentum sequence ( $p \backslash .05)$.

\section{Discussion}

The present study examined the PM perceptions of virtual actors and observers who viewed an audiovisual simulation of positive and negative momentum sequences within a table tennis competition, as well as how their PM perceptions were affected by a sudden interruption. Results 
Fig. 1 PM perceptions according to Momentum Sequence and Involvement Type (a) and according to Momentum Sequence and
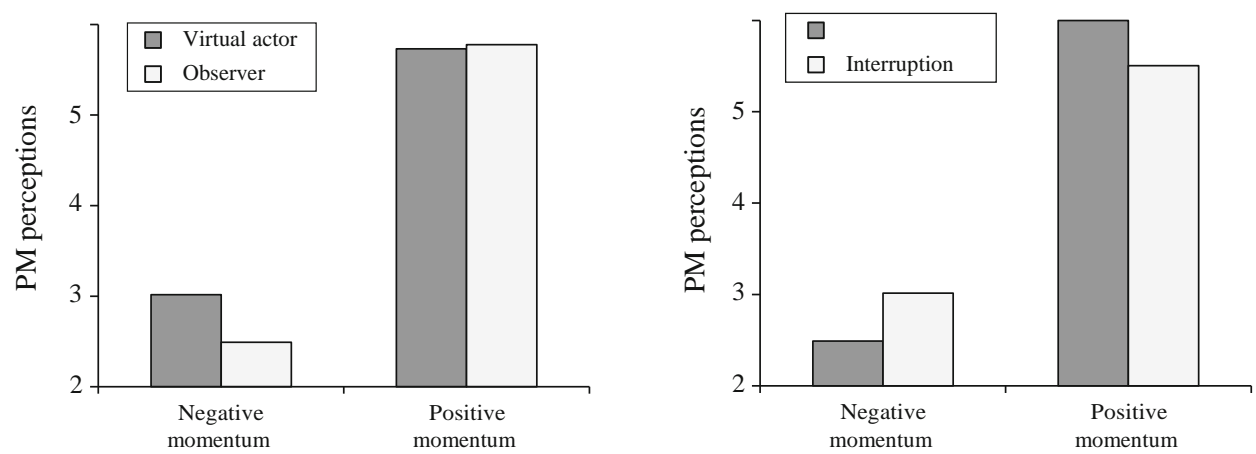

indicated that PM perceptions were higher in the positive momentum condition than in the negative momentum condition, thus supporting previous findings that moving toward (or away from) a goal to be attained has positive (or negative) consequences on PM perceptions (e.g., Eisler and Spink 1998; Kerick et al. 2000; Markman and Guenther 2007; Stanimirovic and Hanrahan 2004; Vallerand et al. 1988).

A main focus of the present study was to examine differences between virtual actors and observers in their PM perceptions when exposed to positive and negative momentum sequences. Following a negative momentum sequence, observers' PM perceptions were lower than virtual actors', indicating that observers expected that the player they were rooting for would be highly affected by the occurrence of negative events. This result is consistent with Deutsch's (1960) finding that observers estimate the momentary feelings of actors to be more extreme than they actually are. However, no differences were found between virtual actors and observers following the positive momentum sequence. Taken as a whole, these results suggest that there is an asymmetry in the way that positive and negative PM are perceived, in the sense that observers are more sensitive than virtual actors with respect to only negative sequences. In part, this asymmetry may be attributable to a combination of observers' proneness to polarize their evaluation of others toward success or failure (Deutsch 1960), and individuals' generally lower sensitivity to positive than to negative outcomes (Baumeister et al. 2001). In other words, the virtual nature of the status of both actors and observers in the present study may have diminished the expected impact of positive momentum sequences because people have been shown to be less sensitive to positive than to negative outcomes. On the other hand, the small but psychologically significant difference between the status of virtual actors and observers in the present study may have been sufficient to elicit differential reactions to negative (and thus more impactful) momentum sequences.
Another main focus was to examine the effects of a sudden interruption on PM perceptions. Regardless of the participants' involvement type (i.e., virtual actor vs. observer), interruptions of positive momentum led to more unfavorable PM perceptions, whereas interruptions of negative momentum led to more favorable PM perceptions. These findings support and extend those of Markman and Guenther (2007), who exposed observers to written positive momentum scenarios. Indeed, the present results indicate that both positive and negative PM experiences are affected by sudden interruptions. Nevertheless, the mean scores for PM perceptions measured after the interruption of positive momentum remained above the midpoint of 4 , whereas mean PM perceptions remained below the midpoint of 4 following the interruption of negative momentum. Thus, although they were clearly affected by the interruption, PM perceptions still remained consistent with the direction of the sequence. This resistance to interruption may be explained by the fact that the sequences involved gradual progress toward victory or defeat. As Markman and Guenther (2007) found, gradual progress toward a goal is perceived as more difficult to disrupt than is momentous progress. Therefore, and employing Adler's (1981) distinction between placid and explosive PM, we speculate that placid PM might be more robust than explosive PM to the impact of sudden interruptions.

The present study presents two limitations. First, although the virtual nature of the simulation facilitated experimental control, such a simulation might have lessened the psychological impact of the momentum manipulations in comparison to a real-life competitive situation. Thus, the comparatively lower psychological impact of the simulation could account for why no significant differences were found between virtual actors' and observers' PM perceptions during a positive momentum sequence, which is known to be less psychologically impactful than is a negative momentum sequence (Briki et al. 2013; Gernigon et al. 2010). Secondly, the manipulation check item we 
employed was specifically designed to assess whether participants had successfully performed the task of imagining being a player (for virtual actors) or a fan (for observers), but not whether participants had successfully performed the task from the actual standpoint of a player or fan. Given that the measurement of actors' and observers' differential PM perceptions was not carried out in a natural setting, it remains possible that the present set of findings may remain specific to the simulation that was used. Consequently, future research might attempt to replicate the present results by enhancing participants' level of involvement.

Given the modest amount of resistance to interruption that was observed in this study, it would seem useful to compare the robustness of placid PM to that of explosive PM. Prior research on PM triggers has not distinguished between placid and explosive PM (e.g., Jones and Harwood 2008), and so it remains to be seen what kinds of situations might naturally trigger placid PM as opposed to explosive PM. It would also be interesting to examine when and how PM could be effectively interrupted. For instance, it would be helpful for coaches and athletes to be able to identify the critical moment at which a timeout would be most likely to disrupt both their own phase of negative PM as well as their opponent's phase of positive PM. In the present research, the interruption of momentum sequences was psychologically neutral (i.e., a power cut). However, coaches' instructions during timeouts have been found to influence the dynamics of athletes' perceptions of control, self-confidence and anxiety (Gernigon et al. 2002). Other psychological processes that are activated during timeouts (e.g., causal attribution, emotional control, self-control, task-focused attention) have been shown to influence athletes' subsequent self-confidence and performance (Gernigon and Delloye 2003). Therefore, it would be fruitful to compare the effects of different psychological strategies of intervention during timeouts on PM dynamics.

Acknowlegment We are grateful to Dorothée Beck, Nidhal M'Saad, and Anthony Sanchez for their technical assistance, Cedric Rouleau for his table tennis specific information, and the table tennis players and students who kindly offered to participate in this research

\section{References}

Adler, P. (1981). Momentum: A theory of social action. Beverly Hills, CA: Sage.

Baumeister, R. F., Bratslavsky, E., Finkenauer, C., \& Vohs, K. D. (2001). Bad is stronger than good. Review of General Psychology, 5, 323-370. doi:10.1037/1089-2680.5.4.323.

Briki, W., Den Hartigh, R. J., Bakker, F. C., \& Gernigon, C. (2012a). The dynamics of psychological momentum: A quantitative study in natural sport situations. International Journal of Performance Analysis in Sport, 12, 573-592.
Briki, W., Den Hartigh, R. J., Hauw, D., \& Gernigon, C. (2012b). A qualitative exploration of the psychological contents and dynamics of momentum in sport. International Journal of Sport Psychology, 43, 365-384.

Briki, W., Den Hartigh, J. R., Markman, K. D., Micallef, J.-P., \& Gernigon, C. (2013). How psychological momentum changes in athletes during a sport competition. Psychology of Sport and Exercise, 14, 389-396.

Brislin, R. W., Lonner, W. J., \& Thorndike, R. M. (1973). Crosscultural research methods. New York: Wiley.

Deutsch, M. (1960). The pathetic fallacy: An observer error in social perception. Journal of Personality, 28, 317-332. doi:10.1111/j. 1467-6494.1960.tb01621.x.

Eisler, L., \& Spink, K. S. (1998). Effects of scoring configuration and task cohesion on the perception of psychological momentum. Journal of Sport and Exercise Psychology, 20, 311-320.

Gernigon, C., Briki, W., \& Eykens, K. (2010). The dynamics of psychological momentum in sport: The role of ongoing history of performance patterns. Journal of Sport and Exercise Psychology, 32, 377-400.

Gernigon, C., \& Delloye, J.-B. (2003). Self-efficacy, causal attribution, and track athletic performance following unexpected success or failure among elite sprinters. The Sport Psychologist, $17,55-76$

Gernigon, C., Yvelin, N., Delignières, D., Ninot, G., \& d'ArripeLongueville, F. (2002). A dynamic approach to the variations in the perception of control, anxiety, and self-confidence, and in their patterns of synchronization during time-out in sport. Journal of Sport and Exercise Psychology, 24, S62.

Goffman, E. (1955). On face-work. Psychiatry, 18, 213-231.

Jones, M. I., \& Harwood, C. (2008). Psychological momentum within competitive soccer: Players' perspectives. Journal of Applied Sport Psychology, 20, 57-72. doi:10.1080/10413200701784841.

Kerick, S. E., Iso-Ahola, S. E., \& Hatfield, B. D. (2000). Psychological momentum in target shooting: Cortical, cognitive-affective, and behavioral responses. Journal of Sport and Exercise Psychology, 22, 1-20.

Markman, K. D., \& Guenther, C. L. (2007). Psychological momentum: Intuitive physics and naive beliefs. Personality and Social Psychology Bulletin, 33, 800-812. doi:10.1177/0146167207301026.

Miller, S., \& Weinberg, R. S. (1991). Perceptions of psychological momentum and their relationship to performance. The Sport Psychologist, 5, 211-222.

Perreault, S., Vallerand, R. J., Montgomery, D., \& Provencher, P. (1998). Coming from behind: On the effect of psychological momentum on sport performance. Journal of Sport and Exercise Psychology, 20, 421-436.

Shaw, J. M., Dzewaltowski, D. A., \& McElroy, M. (1992). Selfefficacy and causal attributions as mediators of psychological momentum. Journal of Sport and Exercise Psychology, 14, 134-147.

Silva, J. M., Cornelius, A. E., \& Finch, L. M. (1992). Psychological momentum and skill performance: A laboratory study. Journal of Sport and Exercise Psychology, 14, 119-133.

Stanimirovic, R., \& Hanrahan, S. J. (2004). Efficacy, affect and teams: Is momentum a misnomer? International Journal of Sport and Exercise Psychology, 2, 43-62. doi:10.1080/1612197X. 2004.9671732.

Taylor, J., \& Demick, A. (1994). A multidimensional model of momentum in sports. Journal of Applied Sport Psychology, 6, $51-70$.

Vallerand, R. J., Colavecchio, P. G., \& Pelletier, L. G. (1988). Psychological momentum and performance inferences: A preliminary test of the antecedents-consequences psychological momentum model. Journal of Sport and Exercise Psychology, $10,92-108$. 